\section{Anforderungen an eine qualifizierte Akutbehandlung Alkoholabhängiger in NRW}

Thomas Kuhlmann ${ }^{1}$, Peter Summa-Lehmann², Gerhard Reymann ${ }^{3}$, Joan Teodor Marcea ${ }^{4}$

${ }^{1}$ Psychosomatische Klinik Bergisch Gladbach

2 Rheinische Landesklinik Düren

${ }^{3}$ Westf. Zentrum f. Psychiatrie, Psychotherapie und Psychosomatik Dortmund

${ }^{4}$ Psychiatrische Klinik Marienborn
Zusammenfassung: Die Fachdiskussion ist seit Anfang der 90er Jahre geprägt von der Entzugsbehandlung und der Integration auch schlecht erreichbarer Subgruppen Drogenabhängiger in qualifizierte Hilfsangebote. Die Erörterung entsprechender Behandlungsangebote für Alkoholabhängige gewinnt erst seit Ende der 90er Jahre an Bedeutung und konzentriert sich auf die unmittelbare Verknüpfung von Entzugs- und motivierender Behandlung mit Integration in die regionale Pflichtversorgung: der qualifizierten stationären Akutbehandlung analog zu den Erfahrungen bei Drogenabhängigen.

Schlüsselwörter: Alkoholabhängigkeit - Qualifizierte Akutbehandlung - Entzug

Demands for a Qualified Clinical Acute Treatment of Alcoholics in NW: Since the early 90's public interest has focussed on detoxication and improvement of therapeutical approach to those drug addicts which are hardly reached by the drug aid system. The discussion as regards alcoholics has been intensified only in the late 90 's. Today the discussion is concentrated on the direct combination of detoxication and motivational therapy within one treatment setting: the qualified clinical acute treatment. These concepts are to be integrated in the regional network for addicts. By this approach all subgroups of alcoholics can be reached and conflicts within the regional aid network can be minimized.

Key words: Alcohol Dependency - Qualified Clinical Acute Treatment - Detoxication

Die Fachdiskussion ist seit Anfang der 90er Jahre geprägt von der Entzugsbehandlung und der Integration auch schlecht erreichbarer Subgruppen Drogenabhängiger in qualifizierte Hilfsangebote. Die Erörterung entsprechender Behandlungsangebote für Alkoholabhängige gewinnt erst seit Ende der 90er Jahre an Bedeutung. Während zunächst unterschiedliche Konzepte für die Entzugs- und Motivationsbehandlung entwickelt wurden, konzentriert sich die aktuelle Diskussion zunehmend auf die unmittelbare Verknüpfung von Entzugsund motivierender Therapie innerhalb einer Behandlung: der qualifizierten stationären Akutbehandlung - analog zu den Erfahrungen bei Drogenabhängigen. Entsprechende Konzepte

Suchttherapie 2001; 2: 93-97

(c) Georg Thieme Verlag Stuttgart · New York ISSN 1439-9903 sind in die regionale Pflichtversorgung zu integrieren, so dass alle Patientengruppen erreicht und Brüche innerhalb des Hilfesystems verringert werden können.

\section{Ausgangssituation in NRW}

Im Februar 1999 wurde das NRW-Landesprogramm gegen Sucht verabschiedet, das nach intensiver Diskussion mit Kosten- und Leistungsträgern, Ärztekammer, Sozialhilfeträgern, Beratungsstellen, Kliniken und Verbänden gemeinsam erarbeitet worden ist. Die qualifizierte stationäre Akutbehandlung Alkoholabhängiger ist ein eigenständiger Behandlungstyp im Sinne dieses Landesprogramms mit folgender Aufgabenstellung:

- Durch einen niederschwelligen Ansatz eine gemeindenahe qualifizierte Behandlung auch derjenigen Alkoholabhängigen zu gewährleisten, die von dem bisherigen stationären Angebot nicht erreicht werden können und für deren Versorgung ambulante Angebote nicht ausreichen,

- durch direkte Einbeziehung in ein regionales Verbundsystem unterschiedlicher psychosozialer Institutionen die Differenzierung und Weiterentwicklung der Suchthilfe vor Ort zu unterstützen und

- durch ein differenziertes und individuumzentriertes Behandlungskonzept sowohl Früherkennung und -behandlung als auch Sekundär- bzw. Tertiärprävention zu ermöglichen, die Motivation zur Annahme weiterer spezifischer Hilfsangebote zu fördern und dadurch zur Begrenzung der aus der Abhängigkeit von Alkohol entstandenen Schäden beizutragen [1].

Die in NRW nahezu flächendeckende Umsetzung des Konzepts der qualifizierten stationären Akutbehandlung für Drogenabhängige hat zu einer deutlich verbesserten Integration vor allem jener Drogenabhängiger in das Drogenhilfesystem geführt, die zuvor kaum erreicht worden sind - chronisch intoxikierte, psychosozial desintegrierte und psychiatrisch komorbide, aber auch junge Drogenabhängige [2-4]. Aufgrund dieser positiven praktischen Ergebnisse und der zahlenmäßig wesentlich größeren Gruppe behandlungsbedürftiger Alkoholabhängiger sollen qualifizierte akutstationäre Angebote, die auf diesem neuen Behandlungsansatz für Alkoholabhängige basieren, flächendeckend in NRW geschaffen und in die regionale Pflichtversorgung integriert werden. Dabei gilt es festzuhalten, dass die bestehenden Alkoholakutstationen vielerorts seit Jahren fachlich qualifizierte Arbeit leisten und weiterhin dringend benötigt werden. Die Fachdiskussion über konzeptionelle Anforderungen an qualifizierte und in ein differenziertes Netzwerk der Suchthilfe eingebundene Akut- 
stationen für Alkoholabhängige ist jedoch bisher deutlich geringer entwickelt als jene über die Behandlung Drogenabhängiger. Da aufgrund ökonomisch geprägter Auseinandersetzungen über die Dauer einer Alkoholentzugsbehandlung fachliche Aspekte zunehmend schwieriger zu vermitteln sind, andererseits Einigkeit über die große Bedeutung der Suchtkrankenbehandlung besteht, hat eine intensive Diskussion zwischen Kliniken, MDK, Kosten- und Leistungsträgern mit gemeinsamen Arbeitstagungen und -gruppen eingesetzt, beginnend in Nordrhein und inzwischen in beiden Landesteilen NRWs, die mit Unterstützung des Landesgesundheitsministeriums zu einem Fachkongress im März 2000 in Münster geführt hat.

\section{Behandlungsansatz (Behandlungsparadigma)}

Die Alkoholabhängigkeit ist eine schwere chronische Erkrankung, die einer fachgerechten Behandlung mit angemessener Berücksichtigung der biopsychosozialen Dimension dieser Krankheit bedarf [5,6]. Eine adäquate Behandlung im Sinne der Primär-, Sekundär- und Tertiärprävention setzt voraus, dass sowohl die Störungen als auch Ressourcen der Betroffenen, die sich auf der psychischen, somatischen und sozialen Ebene diagnostizieren lassen, in die Behandlungsstrategie einbezogen werden. Eine enge Kooperation zwischen ambulanten und teil- bzw. vollstationären Behandlungs- und Betreuungsangeboten ist Voraussetzung sowohl zur Sicherung des Behandlungserfolgs als auch zur Annahme notwendiger Behandlungsangebote.

Die bis Anfang der 90er Jahre zu konstatierende vorherrschende Orientierung der Suchthilfe in Deutschland auf die KOMM-Struktur und das Abstinenzparadigma ist im Grundsatz zwar nach wie vor ein wesentliches Element jeglicher Behandlung, in ihrer ursprünglichen Starrheit jedoch nicht als dem Krankheitsbild angemessen zu betrachten:

Eine enge Kooperation der einzelnen Glieder der regionalen Suchthilfe und die unmittelbare Verzahnung an den jeweiligen Übergängen, vor allem bzgl. Beratungsstellen, qualifizierter Akutbehandlung, Entwöhnungsbehandlung und Selbsthilfegruppen, ist Voraussetzung für den Behandlungserfolg und die Akzeptanz notwendiger und möglichst wohnortnaher Hilfsangebote. Um die heterogene Gruppe Alkoholabhängiger $\mathrm{zu}$ erreichen, ist es entscheidend, unter Berücksichtigung bestehender Störungen die vorhandenen Ressourcen zu aktivieren und jede therapeutische Kontaktmöglichkeit zu nutzen, um das Fortschreiten der chronischen Erkrankung zumindest zu lindern und die mit der Abhängigkeit von Alkohol verbundenen seelischen, körperlichen und sozialen Störungen und Schäden zu überwinden oder zu begrenzen unter aktiver Einbeziehung des Patienten in allen Behandlungsphasen. Die Kooperation mit den therapeutischen Fachdisziplinen und allen Einrichtungen der Suchthilfe und psychosozialen Diensten ist anzustreben. Entsprechend der Heterogenität der Alkoholabhängigen sind diese Ziele weder ausschließlich über ambulante noch über hochschwellige stationäre Angebote zu erreichen $[4,7-11]$.

\section{Zielgruppen}

Die wichtigsten Zielgruppen können wie folgt skizziert werden:

- Einstieg in den Ausstieg: Diese Zielgruppe hatte in der Regel aufgrund permanenter Intoxikation, psychosozialer Desintegration und körperlicher Schäden kaum stabile Kontakte zum Hilfssystem. Deshalb sind Fähigkeit und Motivation zu suchtmittelfreiem Leben Fokus der Behandlung, jedoch keine Voraussetzung zur stationären Aufnahme.

- Ambivalente Ausstiegswillige: Diese Zielgruppe ist charakterisiert durch eine ausgeprägtere Abstinenzorientierung bei noch zwiespältiger innerer Einstellung und entsprechend im Rahmen der Behandlung gezielt in ihrer Motivationsbildung zu unterstützen mit dem Ziel der Einbindung in weiterführende ambulante Betreuungs- und Behandlungsangebote.

- Therapiemotivierte mit Abstinenzwunsch: Bei dieser Zielgruppe ist eine gezielte Vorbereitung auf bereits geplante und vom Leistungsträger bewilligte Entwöhnungsbehandlung durchzuführen, welche bereits zumeist über die Suchtberatungsstellen ambulant vorbereitet worden sind.

Die Mitbehandlung allgemeinpsychiatrischer Störungen bei manifester Alkoholabhängigkeit ist Fokus der Behandlung und Voraussetzung zur Integration und psychosozialen Stabilisierung dieser von Ausgrenzung bedrohten Patientengruppe, sofern das Ausmaß allgemeinpsychiatrischer Störungen nicht in den Vordergrund rückt [12].

Auch die konkrete Einbeziehung wichtiger Bezugspersonen aus dem sozialen Umfeld und die unmittelbare Auseinandersetzung mit Selbsthilfegruppen sind wesentliche Bestandteile der qualifizierten Akutbehandlung und erfordern einen gemeindenahen Kontext.

Manifeste Alkoholabhängigkeit entwickelt sich stets im $\mathrm{Zu}$ sammenwirken unterschiedlicher Faktoren im Sinnes des 3-Faktoren-Modells nach Feuerlein, deren konkrete Ausprägung jedoch von Patient zu Patient erheblich differieren kann. Die therapeutische Grundhaltung dieses skizzierten Behandlungsansatzes ist geprägt von sozialpsychiatrisch orientierter Arbeit und basiert auf dem Leitgedanken der psychodynamisch orientierten, tiefenpsychologisch fundierten Psychotherapie bzw. integrierenden verhaltensmodifizierenden wissenschaftlich anerkannten Therapieformen [10,11,13-15].

\section{Behandlungsziele}

Die qualifizierte stationäre Akutbehandlung Alkoholabhängiger umfasst die somatischen und psychischen Aspekte der Suchterkrankung in ihrer besonderer Ausprägung am einzelnen Patienten. Aufgabe der Behandlung ist die Motivierung und Unterstützung des Patienten in seiner Fähigkeit, die eigene Abhängigkeitserkrankung in ihrer Ausprägung und Funktion zu erkennen und zumindest in Ansätzen alternative Krisenbewältigungsstrategien und ein persönliches Abstinenzkonzept zu entwickeln.

Dies erfordert, Eigen- und soziale Verantwortung zu übernehmen, die subjektiven Belastungsgrenzen zu realisieren und vermehrt einzuhalten, die soziale Realität differenziert 
wahrzunehmen und sich mit Frustration und Bedürfnisbefriedigung auseinander zu setzen.

Ziel der Behandlung ist die Überwindung der manifesten Alkoholabhängigkeit mit der grundsätzlichen Perspektive der Abstinenz und die psychische, physische und psychosoziale Stabilisierung des Patienten. Aufgrund der Rückfallgefährdung als immanentem Bestandteil der chronischen Alkoholabhängigkeit ist die Entwicklung tragfähiger therapeutischer Kontakte auch zu sozial desintegrierten, psychisch labilen und somatisch mehrfach erkrankten chronisch Alkoholabhängigen notwendig. Wiederaufnahmen bei Rückfällen mit bestehender stationärer Behandlungsbedürftigkeit sind nicht nur möglich, sondern gewünscht $[4,8,11,16,17]$.

\section{Indikationen}

Aufgenommen werden Patienten mit manifester Alkoholabhängigkeit, mehrfachem Suchtmittelkonsum bei vorrangiger Alkoholabhängigkeit und schädlichem Gebrauch von Alkohol mit zunehmender Tendenz im Sinne von Frühbehandlung.

Voraussetzung für die Aufnahme ist bestehende Behandlungsnotwendigkeit inklusive PsychKG bzw. Betreuungsrecht und die Bereitschaft, aktuell auf Suchtmittel und Konsumgewohnheiten zu verzichten mit dem Ziel der umfassenden Stabilisierung. Bestehende Kontakte zu psychosozialen Diensten, dem Verbundsystem der Suchthilfe und niedergelassenen Ärzten werden angestrebt und gefördert.

\section{Kontraindikationen}

Nicht aufgenommen bzw. in andere Institutionen verlegt bzw. entlassen werden Patienten, deren somatische Erkrankungen vorrangig sind (z.B. dekompensierte alkoholtoxische Kardiomyopathie etc.) oder deren allgemeinpsychiatrische Erkrankungen Behandlungspriorität (z.B. exazerbierte akute Psychosen) haben. Entscheidend ist die Gesamtkonstellation des Einzelfalls.

\section{Behandlungsdauer}

Die qualifizierte stationäre Akutbehandlung erfordert die Einbeziehung sowohl der somatischen als auch psychischen Probleme des Entzugs und die therapeutische Bearbeitung der bisherigen Entwicklung der Suchterkrankung. Die Motivierung der Patienten zur selbstkritischen Auseinandersetzung mit der eigenen Abhängigkeit, dem Wiederentdecken eigener Ressourcen und zur Annahme weitergehender Hilfsangebote erfordert die Einbeziehung psychischer Störungen nach Abklingen somatischer Entzugssymptome in die Behandlung. Ausmaß der Intoxikation, Konsumverhalten vor Aufnahme, Entzugsverlauf und Auftreten somatischer und psychischer Komplikationen prägen den Behandlungsverlauf entscheidend und individuell unterschiedlich und erfordern durchschnittlich eine stationäre Behandlungsmöglichkeit von bis zu drei Wochen zur Restitution der Kognitionsfähigkeit [18]. Dieser zeitliche Rahmen ist indikationsabhängig.

\section{Diagnostik}

Die fachgerechte Abklärung des Ausmaßes der Abhängigkeitserkrankung und ihrer individuellen Ausprägung erfordert differenzierte diagnostische Maßnahmen:

- allgemeinmedizinische Untersuchung,

- ärztliches Gespräch inkl. psychiatrischer Diagnostik und Erhebung der Suchtanamnese,

- Eigen-, Sozial- und Pflegeanamnese,

- Labordiagnostik einschließlich Drogenscreening sowie bei Bedarf apparativer Zusatzdiagnostik (Röntgenthorax, EKG, EEG, Sonographie etc.),

- nach Indikation und Zustand des Patienten weitere Diagnostik,

- bei Bedarf psychologische Testuntersuchungen

- im weiteren Verlauf stichprobenartige Drogenscreenings (Urinabgabe unter Sicht, Atemalkohol),

- bei Bedarf konsiliarärztliche Untersuchungen [7].

\section{Behandlungsplanung und -verlauf}

Nach den Ergebnissen der Aufnahmeuntersuchungen wird ein individueller Behandlungsplan im Rahmen des therapeutischen Standardprogramms erstellt und mit dem Patienten auf dem Hintergrund einer umfassenden und differenzierten Anamnese eine Zielhierarchie vereinbart, die im Behandlungsverlauf überprüft und weiterentwickelt wird. Unverzichtbare Grundlage der Behandlung ist einerseits die Abstinenz von Alkohol und anderen psychotropen Substanzen, andererseits die Einbeziehung in das regionale Netzwerk der Suchthilfe einschließlich regionaler Pflichtversorgung. Die aktive Teilnahme des Patienten an der Behandlung hängt wesentlich vom Ausmaß der Intoxikation, dem Detoxikationsverlauf und den somatischen und psychischen Störungen ab. Der Behandlungsplan umfasst:

1. die Überwindung somatischer Intoxikations- und Entzugssymptome einschließlich diagnostischer Abklärung somatischer und psychischer Störungen,

2. die therapeutische Bearbeitung im Einzelkontakt und der Gruppe (psychiatrisch-psychotherapeutisch, pflegerisch, sport- und kreativtherapeutisch) bei vorrangiger Binnenorientierung und

3. die Vertiefung des therapeutischen Prozesses mit zunehmender Außenorientierung, Vorbereitung möglicher Anschlussbehandlungen und Einbeziehung des sozialen Umfeldes während der Behandlung [3,4,7,9,16,18-21].

Nach Abschluss der ersten beiden Teilbereiche werden diejenigen entlassen bzw. verlegt, deren Perspektive bereits vor Behandlungsbeginn sichergestellt war (z.B. Entwöhnungsbehandlung, ambulante Nachbetreuung etc.) oder die einer Intensivierung des „Einstiegs in den Ausstieg“ noch nicht zugänglich sind und eines anderen Settings bedürfen.

Jene Suchtkranken, die aufgrund psychischer Labilität, somatischer Erkrankungen, sozialer Desintegration oder ausgeprägter Ambivalenz gegenüber einer bevorstehenden Entwöhnungsbehandlung im besonderen Maße gefährdet sind, bedürfen einer weitergehenden, im dritten Teilbereich skizzierten stationären Behandlung. Dabei wird besonderes Gewicht gelegt auf die Förderung selbstkritischer Reflexion suchttypischer Verhaltensweisen, die Übernahme von mehr Eigen- und sozialer Verantwortung und erste Umsetzungs- 
schritte zur alternativen Bewältigung krisenhafter Situationen.

Grundlage der Behandlung sind das gruppenorientierte therapeutische Programm im Tages- und Wochenablauf und der therapeutische Einzelkontakt mit den Mitarbeitern des multiprofessionellen Teams. Die Teilnahme am gesamten Behandlungsangebot ist verpflichtend. Aufgrund der Komplexität der zu behandelnden Störungen und Krankheitsbilder ist die Notwendigkeit der Teilnahme an den einzelnen Behandlungsangeboten stets von neuem zu prüfen unter Berücksichtigung sowohl der Dynamik der Patientengruppe als auch der besonderen Situation des einzelnen Patienten.

Wesentliche therapeutische Problemstellungen werden in der Regel erst mit Abklingen des körperlichen Entzugssyndroms deutlich, wenn die Patienten ihre innere Zerrissenheit und somatische Beeinträchtigung als qualvoll und bedrohlich wahrnehmen und die eigene Hilfsbedürftigkeit nur schwer $\mathrm{zu}$ akzeptieren vermögen. In dieser noch sehr labilen Behandlungsphase sind die Kontinuität des Behandlungsrahmens und ein Behandlungssetting erforderlich, welches die Ängste, Widerstände und intrapsychischen Konflikte dieser Zielgruppe berücksichtigt und motivationsfördernde therapeutische Angebote vorhält $[8,12,18]$. Während des psychopathologischen Postdetoxikationssyndroms bedürfen die Patienten besonderer Zuwendung, klarer Strukturen und eines Behandlungsrahmens, in dem sie ihre inneren Spannungen, jedoch auch ihre psychosozialen Schwierigkeiten und physischen Probleme wahrnehmen, ausagieren und bearbeiten können. Die Motivation zur Annahme weiterführender Hilfsangebote wie z.B. ambulante oder stationäre Entwöhnungstherapie wird in dieser Behandlungsphase wesentlich geprägt und gefördert, die bewusste Wahrnehmung und Auseinandersetzung mit den somatischen und psychischen Aspekten des Entzugs begünstigt die Akzeptanz der eigenen Hilfsbedürftigkeit und die Bereitschaft zu suchtmittelfreiem Leben [15,21]. Eine starre, fachlich nicht $\mathrm{zu}$ rechtfertigende Begrenzung der Behandlungsdauer verhindert die Überwindung dieser von psychischer Instabilität geprägten Phase, erhöht die Rückfallneigung durch vorzeitigen Behandlungsabbruch und erschwert die Akzeptanz zur Annahme weiterführender Hilfsangebote $[7,11,15,16,21]$.

Die Behandlung kann vollstationär, je nach individuellem Verlauf jedoch auch teilstationär durchgeführt oder beendet und ambulant fortgesetzt werden.

\section{Personelle Ausstattung/Behandlungsplan}

Die qualifizierte Akutbehandlung alkoholbedingter Erkrankung ist Bestandteil der psychiatrischen Versorgung und bedarf eines multidisziplinären Teams und eines differenzierten Behandlungsplans, der:

- allgemeinmedizinische und psychiatrische Behandlung inklusive Gesundheitserziehung,

- psychotherapeutische Interventionen mit Einzel- und Gruppensitzungen,

- pflegerische und sozialarbeiterische Interventionen,

- Entspannungstraining, Bewegungs- und Sporttherapie,

- Beschäftigungs- und Kreativtherapie,

- Stationsversammlung und bei Bedarf Krisensitzungen,
- Übernahme von Eigen- und sozialer Verantwortung der Patienten in allen Lebensbereichen und - rückfallbezogene Interventionen umfasst.

Die Einbeziehung in die regionale Pflichtversorgung im Rahmen differenzierter regionaler Verbundnetze ist anzustreben. Die Akutstation selbst bedarf eines äußeren Rahmens, der kontrollierte Behandlungsbedingungen (z.B. Kontrolle wegen Suchtmittelmissbrauch) sicherstellt: je nach konkreten Bedingungen vor Ort offen, geschlossen oder z. B. fakultativ offen. Die personelle Besetzung richtet sich qualitativ und quantitativ nach der PsychPV (je 50\% Fallgruppe S1 und S2).

Für eine 20-Betten-Station mit einer Durchschnittsbelegung von 18 Patienten ergibt sich z.B.

- 12 pflegerische Mitarbeiter/-innen,

- 2,5 Ärzte einschließlich Oberarzt (mit psychiatrisch-psychotherapeutischer FA-Qualifikation),

- 0,5 Dipl.-Psychologen,

- 1,5 Sozialarbeiter/-innen,

- 0,5 Ergotherapeuten/-innen,

- 0,5 Bewegungstherapeuten/-innen.

Kontinuierliche externe Supervision für alle klinischen Mitarbeiter sowie kontinuierlicher Erfahrungsaustausch und Wissensvermittlung neben den notwendigen Fort- und Weiterbildungsmaßnahmen sind unverzichtbar.

\section{Kostenträgerschaft}

Die qualifizierte stationäre Akutbehandlung Alkoholabhängiger dient der Überwindung der manifesten Alkoholabhängigkeit bzw. der Besserung oder Linderung des Ausmaßes der Alkoholabhängigkeit. Kostenträger dieser Behandlung sind die Krankenkassen.

\section{Qualitätssicherung}

Die fachgerechte und patientenorientierte Durchführung der qualifizierten stationären Akutbehandlung erfordert einen ständigen innerklinischen Prozess zur Überprüfung der erreichten Qualität und Weiterentwicklung des Behandlungskonzepts entsprechend den sich verändernden Bedingungen des Gesundheits- und Sozialsystems und den Veränderungen der Patienten.

Die Strukturqualität wird über die Basisdokumentation sichergestellt und über die Dokumentation der

- eingehenden psychiatrischen Diagnostik und Anamnese,

- körperlichen Untersuchung und Labor- sowie konsiliarärztlichen Befunde,

- pflegerischen und sozialarbeiterischen Befunde,

- Krankenblattführung einschließlich Dokumentation der Einzel- und Gruppenkontakte.

Die Prozesskategorie der Qualitätssicherung umfasst alle den Behandlungsverlauf eines Patienten betreffenden Maßnahmen. Sicherstellung und Weiterentwicklung von Struktur-, Prozess- und Ergebnisqualität erfordern enge, interdisziplinäre und patientenorientierte Zusammenarbeit aller Mitarbeiter und Berufsgruppen des einzelnen Teams, aber auch der gesamten Klinik. 


\section{Stellung im Suchthilfenetz}

Die Stationen zur qualifizierten Akutbehandlung Alkoholabhängiger müssen in differenzierte regionale Verbundnetze der Suchthilfe eingebunden werden; eine enge Kooperation mit allen Institutionen des Gesundheitswesens (psychiatrische Fachkrankenhäuser, somatische Fachdisziplinen und niedergelassene Ärzte) ist dringend erforderlich.

Unter diesen Voraussetzungen ist die Einbeziehung dieser Stationen in die regionale Pflichtversorgung Alkoholabhängiger unabdingbar. Dabei sind entsprechende Regelungen vor Ort einschließlich Berücksichtigung regionaler Besonderheiten erforderlich.

\section{Literatur}

${ }^{1}$ Ministerium für Frauen, Jugend, Familie und Gesundheit des Landes NRW Landesprogramm gegen Sucht, MFJFG, 40190, Düsseldorf, 2/99

${ }^{2}$ Klein M. 1. Zwischenbericht zum Modellprojekt „Behandlung sofort Köln“ 7/1997. Kath. Fachhochschule NRW, Abt. Köln. 50668 Köln

${ }^{3}$ Kuhlmann T, Hasse HE, Sawalies D. Die qualifizierte stationären Akutbehandlung Drogenabhängiger in NRW. Psychiatrische Praxis. Stuttgart-New York: Georg Thieme Verlag, 1994; 21: $13-18$

${ }^{4}$ Kuhlmann T, Hasse HE, Sawalies D. Harm-Reduction und niederschwellige Drogenhilfe in NRW - über die Anforderung an eine qualifizierte stationäre Akutbehandlung Drogenabhängiger Sucht 40. Geesthacht: Neuland-Verlagsgesellschaft mbH, 1994; Jahrgang, Heft 1: 50-56

${ }^{5}$ Feuerlein W. Alkoholismus-Mißbrauch und Abhängigkeit. Stuttgart-New York: Thieme Verlag, 1989; 4. überarbeitete Auflage

${ }^{6}$ Kielholz P, Ladewig D. Drogenabhängigkeit des modernen Menschen. München: Lehmann, 1972

${ }^{7}$ Banger M, Summa-Lehmann P, Schäfer R. Akutbehandlung Alkoholabhängiger in psychiatrischen Kliniken. Rheinisches Ärzteblatt. Düsseldorf, 10/98: 20-23

${ }^{8}$ Driessen M, Veltrup C, Junghanns K, Przywara A, Dilling H. Kosten-Nutzen-Analyse klinisch-evaluierter Behandlungsprogramme. Nervenarzt 1999; 70: 463-470

${ }_{9}^{9}$ Aktion psychisch Kranke: Gemeindepsychiatrische Suchtkrankenversorgung - regionale Vernetzung medizinischer und psychosozialer Versorgungsstrukturen. In: Jagoda B, Kunze H (Hrsg). Aktion psychisch Kranke. Graurheindorfer Str. 15, Bonn; Band 21

${ }^{10}$ Mann K, Buchkremer G. Die pharmakologische Rückfallprophylaxe bei Alkoholabhängigen - Bedarf und Möglichkeiten In: Mann K, Buchkremer G (Hrsg). Sucht - Grundlagen, Diagnostik, Therapie. Stuttgart, Jena, New York: Fischer-Verlag: 317-321

${ }^{11}$ Summa-Lehmann P. Qualifizierte Akutbehandlung Alkoholabhängiger. Tagungsband des Landschaftsverbandes WestfalenLippe im Druck, 2001

12 Schwoon D, Krausz M. Psychose und Sucht - Krankheitsmodelle, Verbreitung, therapeutische Ansätze. Freiburg i.b.: LambertusVerlag, 1992

${ }^{13}$ Die psychoanalytisch-interaktionelle Methode. In: Heigl-Evers A, Ott J (Hrsg). Göttingen, Zürich: Vandenhoeck und Ruprecht, 1995; 2. Auflage

${ }^{14}$ Kuhlmann Th. Harm reduction - zum Paradigmenwechsel in der Drogenarbeit. Psychiatrische Praxis. Stuttgart, New York: Thieme-Verlag, 1996; Heft 4: 157-160
${ }^{15}$ Summa-Lehmann P. Alkoholabhängig? - die therapeutische Gemeinschaft als unsere sozialpsychiatrische Antwort. in Moderne Psychiatriegeschichte: Janssen GmbH Neuss, 1989: 167-180

${ }^{16}$ Beutel M (Hrsg.). Bundesverband für stationäre Suchtkrankenhilfe: Motivation in der Suchttherapie - intrapsychischer Prozeß und versorgungspolitische Aufgabe. Geesthacht: Neuland-Verlagsgesellschaft $\mathrm{mbH}, 1998$

${ }^{17}$ Körkel J, Lauer G, Scheller R. Sucht und Rückfall. Stuttgart: Enke Verlag 1995, 1995

${ }^{18}$ Horak M, Soyka M. Restitution neuropsychologischer Defizite von Alkoholkranken in der Phase der frühen Abstinenz: Ergebnisse einer neuropsychologischen Studie. Sucht 1999; 45: 376-389 (6)

${ }^{19}$ Reymann G, Erdmann A. Ergotherapie in der qualifizierten Akutbehandlung Alkoholabhängiger. praxis ergotherapie Oktober 1999; Jg. 12 (Heft 5): 399-400

${ }^{20}$ Stetter F, Mann K. Zum Krankheitsverlauf Alkoholabhängiger nach einer stationären Entgiftungs- und Motivationsbehandlung. Nervenarzt 1997; 68: 574-581

${ }^{21}$ Wienberg G. Die vergessene Mehrheit - zur Realität der Versorgung alkohol- und medikamentenabhängiger Menschen. Psychiatrieverlag Bonn, 1992

Dr. med. Thomas Kuhlmann

Psychosomatische Klinik Bergisch-Gladbach

Schlodderdicher Weg 23a

51469 Bergisch-Gladbach 